\title{
The 2000 revision of the Declaration of Helsinki: a step forward or more confusion?
}

\author{
Heidi P Forster, Ezekiel Emanuel, Christine Grady
}

\begin{abstract}
At a time when there was great attention and intense public controversy surrounding clinical (especially multinational) research, the 52nd general assembly of the World Medical Association (WMA) adopted the 5th revision of the Declaration of Helsinki (in October, 2000) - available at www.wma.net. These revisions are the most substantial adaptations since those adopted by the 29th WMA assembly in October, 1975. The commitment to revision of the declaration acknowledged that deficiencies and disagreements in interpretation needed to be corrected and that ethical standards evolve. Nevertheless, this revision process resulted in a controversial version of the declaration. Reports on the revisions have focused mainly on clinical trials that use placebos; ; ${ }^{1,2}$ but because of the role of the Declaration of Helsinki in the ethics of research, a more thorough examination is needed. Here, we analyse the process of revision and the major changes made to the declaration.
\end{abstract}

\section{Process of revision}

Since 1997, much debate on the ethics of trials, especially trials in the developing world of regimens to prevent vertical transmission of HIV, has taken place. Most commentators agreed that changes to the declaration were needed, especially clarification of the placebo control issue., ${ }^{2,3}$ Several drafts were considered, including one that was rejected at the WMA meeting in April, $1999 .{ }^{4}$ Afterwards, a new four-member committee began work on another version. The committee was composed of clinicians, who represented national professional medical organisations from more-developed countries; none of these clinicians was recognised as having expertise in the conduct of clinical research or bioethics. Although the need for, and direction of the revisions was publicly debated for more than a year before the latest revision, ${ }^{5}$ only 2 weeks were made available for comment and critique between the appearance of the final proposed version and the assembly vote. This secretive and rushed process, without much time for comment on the content and wording of changes, has been criticised as conflicting with the declaration's own spirit of transparency and disclosure. ${ }^{6,7}$ Such a process might account for imprecise wording, conflicting interpretations, and alienation of those who were excluded from comment, thereby compromising the integrity of the document.

In the Spring of 2001, the WMA announced the development of a working group to revisit certain provisions ${ }^{8}$ specifically the controversial ${ }^{2,9}$ placebo control guideline. Perhaps in response to criticism about the previous revision process, the WMA states that it plans a comprehensive study and wide consultation", suggesting a more thorough and inclusive process.

Lancet 2001; 358: 1449-53

Department of Clinical Bioethics, Warren G Magnuson Clinical Center, National Institutes of Health, Bethesda, MD, USA (H P Forster JD, E Emanuel MD, C Grady PhD)

Correspondence to: Dr Christine Grady, National Institutes of Health, Department of Clinical Bioethics, Building 10, Room 1C118, Bethesda, MD 20892, USA (e-mail: cgrady@nih.gov)

\section{Changes to declaration}

In major alterations to the declaration's tone, the WMA asserts a change in its status and authority. Previously, the WMA called the declaration "recommendations" to physicians that "should be kept under review ... [as] a guide" and stated that "Physicians are not relieved from criminal, civil, and ethical responsibilities under the laws of their own countries" (panel 1). The revision states that the declaration no longer contains "recommendations" but rather is "a statement of ethical principles", and now claims priority over national laws and regulations (panel 1).

In 1964, when ethical guidance on research in human beings was mainly restricted to the Nuremberg Code and most countries did not have relevant laws and regulations, the declaration could legitimately have claimed supreme authority. But in 2001, many sources of ethical guidance on human research exist, including general guidelines ${ }^{10-12}$ and guidelines for specific types of research (eg, HIV preventive vaccine research ${ }^{13}$ ), and many countries now have their own laws and regulations governing research in human beings. Greg Koski, director of the US Office for Human Research Protections, acknowledged the differences between US law and the new declaration: "I don't believe that we would ... take the declaration as the literal basis for new regulations". ${ }^{14}$ The conflicts and inconsistencies between such documents and laws, and within the declaration itself are not addressed by the 2000 version. Rather, it claims to be definitive guidance. The authority and practical meaning of this self-proclaimed status is unclear.

\section{Modification of structure}

Previous versions of the declaration had four parts: introduction; basic principles; medical research and professional care; and non-therapeutic biomedical research with human beings. In the 2000 declaration, the nontherapeutic research category was eliminated. Although such categorisation had been severely criticised as inaccurate, illogical, and ignoring the possibility of some types of research, it did accentuate the widely accepted fact that research without the prospect of direct benefit to individual participants-non-therapeutic research-is ethically justifiable in certain circumstances..$^{15}$ Removal of this section could add to the therapeutic misconception by not clearly distinguishing between research and clinical care. $^{16}$ The elimination of principles governing people 


\section{Panel 1: Selected major changes in wording to the declaration}

\section{Topic}

Authority

\section{6 version}

Physicians are not relieved from criminal, civil and ethical responsibilities under the laws of their own countries. (Introduction, last paragraph)

Study design;

In every medical study, every patient-including those of a control group, if any-should be assured of the best proven diagnostic and therapeutic method. (II.3)

Risk analysis
Physicians should abstain from engaging in research projects involving human subjects unless they are satisfied that the hazards involved are believed to be predictable. Physicians should cease any investigation if the hazards are found to outweigh the potential benefits. (I.7)

\section{0 version}

No national ethical, legal, or regulatory requirement should be allowed to reduce or eliminate any of the protections for human subjects set forth in this declaration. (Provision 9)

The benefits, risks, burdens, and effectiveness of a new method should be tested against those of the best current prophylactic, diagnostic and therapeutic methods. (Provision 29)

Physicians should abstain from engaging in research projects involving human subjects unless they are confident that the risks involved have been adequately assessed and can be satisfactorily managed. Physicians should cease any investigation if the risks are found to outweigh the potential benefits or if there is conclusive proof of positive and beneficial results. (Provision 17) enrolled in research without intention for, or prospect of, direct benefit (as is common in epidemiology, natural history, phase I trials, and other research) could imply that such research is not ethical. The revised declaration does indicate that it can be appropriate to enroll "healthy volunteers" (provision 16)-implicitly endorsing the idea that benefit to participants is not an absolute requirement of ethical research. Nonetheless, the overarching tone of the declaration and several specific provisions $(5,19,24,28$, 29) suggest that benefit to participants is necessary for research to be ethical.

\section{Improvements}

Several of the revisions are clear improvements on previous versions. First, the document was updated to provide new guidance on ethical obligations for monitoring and oversight of clinical research. In addition to independent review of research protocols, committees (institutional review boards or regional ethics committees) are afforded the "right to monitor ongoing trials". Also, investigators have an "obligation to provide monitoring information, especially any serious adverse events" (provision 13; panel 2). Although emphasis on the importance of

\section{Panel 2: Selected potentially controversial additions to the declaration \\ Topic \\ Specific 2000 version \\ provision}

Conflicts

of interest

13

Monitoring

and oversight

Post-study

access

Benefit

requirement

Vulnerable

populations

Research with people unable to consent: necessity requirement and emergency research

Research with 24 minors: necessity requirement
The researcher should also submit to the committee, for review, information regarding funding, sponsors, institutional affiliations, and other potential conflicts of interest.

Each potential subject must be adequately informed of the aims, methods, sources of funding, any possible conflicts of interest, institutional affiliations of the researcher, the anticipated benefits and potential risks of the study and the discomfort it may entail.

Sources of funding, institutional affiliations and any possible conflicts of interest should be declared in the publication.

The committee has the right to monitor ongoing trials. The researcher has the obligation to provide monitoring information to the committee, especially any serious adverse events.

At the conclusion of the study, every patient entered into the study should be assured of access to the best proven prophylactic, diagnostic and therapeutic methods identified by the study.

Medical research is only justified if there is a reasonable likelihood that the populations in which the research is carried out stand to benefit from the results of the research.

Some research populations are vulnerable and need special protection. The particular needs of the economically and medically disadvantaged must be recognised. Special attention is also required for those who cannot give or refuse consent for themselves, for those who may be subject to giving consent under duress, for those who will not benefit personally from the research, and for those for whom the research is combined with care.

26 Research on individuals from whom it is not possible to obtain consent, including proxy or advance consent, should be done only if the physical/mental condition that prevents obtaining informed consent is a necessary characteristic of the research population. The specific reasons for involving research subjects with a condition that renders them unable to give informed consent should be stated in the experimental protocol for consideration and approval of the research committee.

These groups should not be included in research unless the research is necessary to promote the health of the population represented and this research cannot instead be performed on legally competent persons. 
monitoring clinical trials rather than just prospectively approving them is desirable and welcomed, phrasing it as a "right" seems to make monitoring optional rather than necessary for each trial. Such wording might be a result of the hasty process rather than the intention of the assembly.

Second, the declaration now recognises the growing concern about financial conflicts of interest for researchers and the use of financial incentives for subjects. However, it adopts only one specific solution to the problemdisclosure of conflicts to review committees (provision 13), participants (provision 22), and publishers (provision 27; panel 2). Although many organisations have adopted disclosure as necessary to management of conflicts of interest, there is no evidence that disclosure alone is either sufficient or effective in protection of research integrity and prevention of harm to participants. Other safeguards, such as prohibiting researchers from having any other financial links (including consultancies, honoraria, ownership in non-public companies, and stocks or options in public companies) with the companies that sponsor their research could be more effective and preferable. Such prohibitions seem to be evolving as the ethical norm. ${ }^{17,18}$ More importantly, independent review committees are unlikely to be so structured or constituted so as to manage the disclosures. Many institutional review boards report enormous workloads, ${ }^{19}$ and might not have either the expertise or the time to properly manage disclosure of conflicts of interest. So although it is a positive advance that the declaration addresses conflicts of interest, its endorsement of disclosure as the solution seems imprudent.

A third important addition is the requirement that at the conclusion of a trial, "every patient entered should be assured of access to the best proven . . . methods identified by the study" (provision 30; panel 2). Much recent debate has stemmed from the fact that in many studies, provisions are not in place to assure participants access to products that are proven effective through their participation in the trial..$^{20}$ Removal of an effective intervention when a study is over, especially in situations in which the intervention is otherwise unavailable to the individual, could not only be harmful, but might also contravene ethical obligations created by engaging people in research. ${ }^{21}$ Unfortunately, how to assure access, and whose responsibility it is, are challenges left unanswered by the 2000 declaration.

The idea of being responsive to communities in which research is done (provision 19; panel 2) reiterates a requirement first stated in the Council for International Organizations of Medical Sciences (CIOMS) international ethical guidelines, ${ }^{10}$ and is a valuable addition. Unfortunately, the language chosen in the 2000 declaration is vague and thus replicates the controversy surrounding the CIOMS language, rather than advancing the issue. Provision 19 supports the position that to avoid exploitation, the research question and results should have relevance to and potential benefit for the population from which participants are drawn..$^{22}$ However, it seems overly narrow and unjustifiable to restrict the notion of benefit to populations participating in research to benefits derived from the results of the research. A broader notion of benefits to the populations would recognise the benefits from the conduct of research (such as capacity development) and not restrict them to those linked solely with results.

Three additional examples of improvements to the declaration merit attention. First, the WMA recognises that society should not be satisfied with present interventions and has an ethical obligation to study new ways to understand, prevent, diagnose, and treat illness and find interventions that are more effective, have fewer side- effects, and are accessible to more people (provision 6; panel 3). Second, in provision 22 (panel 3), the WMA affirms that written informed consent is neither ethically required nor feasible in every circumstance and culture. ${ }^{23}$ Finally, the previous declaration stated that trials should be stopped if the "hazards are found to outweigh the potential benefits" (provision 1.7; panel 1). In an effort to accord the declaration with current ethical practice, the new version states that trials should also be stopped "if there is conclusive proof of positive and beneficial results" (provision 17; panel 1).

\section{Concerns}

One drawback in the new revision relates to vulnerable populations-groups in need of additional safeguards because they cannot protect their own interests through valid informed consent. ${ }^{8}$ Examples of populations traditionally regarded as vulnerable include people with mental impairments that affect their capacity to make decisions, people in institutions, and children. Although the previous version stated that "particular caution" should be used with patients in a dependent relationship with their physician (provision 1.10) and made special recommendations for cases of legal incompetence (provision 1.11), the new declaration goes further, making every conceivable person vulnerable, from patients with an illness, to those who cannot give consent, to healthy volunteers (provision 8; panel 2). The new declaration expands the category of vulnerability so broadly that it eliminates this category as a special protection; if everyone is vulnerable, no one is entitled to special protection.

Provision 17 addresses the issue of predictable risk by stating that physicians should not do research in human beings unless risks have been adequately assessed and can be satisfactorily managed (panel 1 ). The meaning of this

\section{Panel 3: Selected additions to the declaration that account for widely-accepted standards}

\begin{tabular}{|c|c|c|}
\hline Topic & Provision & 2000 version \\
\hline $\begin{array}{l}\text { Scope of } \\
\text { research in } \\
\text { human beings }\end{array}$ & 1 & $\begin{array}{l}\text { Medical research involving human } \\
\text { subjects includes research on } \\
\text { identifiable human material or } \\
\text { identifiable data. }\end{array}$ \\
\hline $\begin{array}{l}\text { Informed } \\
\text { consent }\end{array}$ & 22 & $\begin{array}{l}\text { After ensuring that the subject } \\
\text { has understood the information, } \\
\text { the physician should then obtain } \\
\text { the subject's freely-given } \\
\text { informed consent, preferably in } \\
\text { writing. If the consent cannot be } \\
\text { obtained in writing, the non- } \\
\text { written consent must be formally } \\
\text { documented and witnessed. }\end{array}$ \\
\hline $\begin{array}{l}\text { Investigator } \\
\text { obligation }\end{array}$ & 10 & $\begin{array}{l}\text { It is the duty of the physician in } \\
\text { medical research to protect the } \\
\text { life, health, privacy, and dignity of } \\
\text { the human subject. }\end{array}$ \\
\hline $\begin{array}{l}\text { Clinical } \\
\text { research } \\
\text { identification }\end{array}$ & 31 & $\begin{array}{l}\text { The physician should fully inform } \\
\text { the patient which aspects of the } \\
\text { care are related to the research. }\end{array}$ \\
\hline Scientific & 6 & $\begin{array}{l}\text { Even the best proven prophylactic } \\
\text { diagnostic, and therapeutic } \\
\text { methods must continuously be } \\
\text { challenged through research } \\
\text { for their effectiveness, efficiency, } \\
\text { accessibility and quality. }\end{array}$ \\
\hline
\end{tabular}


provision is unclear since in some studies, such as in those of phase-I safety trials, the objective is to assess risks and endpoints, and unpredictable risks are always possible. A literal interpretation of the declaration's wording seems to preclude such studies, since not all risks can be prospectively assessed.

In provision 24 (panel 2) the 2000 declaration states that research should not include people who are legally incompetent, incapable of giving consent, or under the age of majority unless necessary to promote the health of such a group and not otherwise attainable with legally competent people. Such a statement might preclude important research-for example, any research with healthy children is unlikely to be considered necessary to promote their health. This provision contravenes other guidelines; CIOMS allows research on healthy minors provided that risk is minimum, ${ }^{8}$ and US guidance mandates that children be included in research unless there is good scientific or ethical cause for their exclusion. ${ }^{24}$ These inconsistencies might be another result of hastily crafted wording with the effect of stifling beneficial research on children and others who cannot provide their own consent.

Several provisions seem to conflict. Provision 19 introduces a benefit requirement for populations in which research is done (panel 2). This requirement is in direct contrast to provision 16, which states that healthy volunteers are allowed to participate. Similarly, provision 20 requires that "subjects must be volunteers and informed participants in the research project". Taken literally, this contradicts provisions 24-26, which address the possibility of proxy consent for people who are incapable of giving consent-people who are neither volunteers nor informed participants. Presumably these provisions were intended to modify provision 20. However, as phrased they remain contradictory.

\section{Placebo controls}

The debate about the appropriate standard of care in research, and especially use of placebo controls when effective therapy exists, was the impetus for the current revision. Substantial debate centered on provision II.3 in the 1996 version, which required that in every medical study all participants "be assured the best proven diagnostic and therapeutic method" ${ }^{2,5,25}$ (panel 1). Criticised as being imprecise and logically inconsistent with randomised trials, the main controversy focused on whether "best proven" should mean the best proven therapy available anywhere in the world or the best-proven available in the region where the study was being done. The new provision 29 (panel 1) replaces "best proven methods" with "best current . . . methods", but the change in wording does not clarify whether use of a host country's standard of care is acceptable when another, possibly more effective intervention is used in another country. Public comments by the Secretary General of the WMA suggest that the drafting committee intended to refer to current methods available anywhere in the world, ${ }^{9}$ but the phrasing of the provision obfuscates the committee's intent. Furthermore, as worded, provision 29 excludes the possibility of various trial designs that are ethically sound. For example, add-on trials are designed to compare placebo with a study agent in participants also receiving standard therapy. Such a trial would be contrary to this provision since it is neither testing a "new method . . . against . . . the best current . . . method" nor restricting "the use of placebo ... [to] studies where no proven . . . method exists". ${ }^{26}$

Most importantly, provision 29 seems to contravene notions of justice. Freedman ${ }^{27}$ argued that there were five situations in which placebos could be justified, including "conditions whose validated optimal treatment is not made freely available to patients because of cost constraints or otherwise". Societies decide how to allocate resources for medical interventions because of competition with other social goods. Because of these societal choices, people are not entitled to every effective medical intervention. ${ }^{28-30}$ In these circumstances, where no entitlement exists, Freedman $^{27}$ claims that withholding treatment "from the placebo cohort does not violate their right to treatment, and may consequently be ethically justified." The new declaration seems to suggest that participants in research studies are entitled to every treatment available anywhere in the world irrespective of national priorities. This is an example of globalisation that could conflict with fair allocation of resources in a country, ultimately making results of research less relevant or useful in certain countries. $^{27,30,31}$

\section{Implementation of the declaration}

The Declaration of Helsinki is an influential source of guidance that assists researchers in the design and implementation of ethical research in human beings. It is encouraging that the declaration is not seen as a timeless pronouncement of ethical ideals but rather as an ongoing document that can be reviewed and regularly revised. The incorporation of current thought, circumstances, and changing ethical ideals is valuable. Although laudable in many respects, the most recent revision of the Declaration of Helsinki is imprecise and ambiguous, possibly the result of a flawed drafting process. The lack of clarity in the declaration and its assertion of supremacy over other guidance and laws creates a practical dilemmaresearchers, review committees, and publishers will have to decide whether particular research complies with the declaration, and whether national or local laws violate the declaration. In effect, either the declaration will be ignored, or its provisions will not be applied uniformly. The US Food and Drug Administration recently published its decision not to incorporate the new declaration into its regulations governing clinical studies in foreign countries. Instead, the Food and Drug Administration will accept studies of new drugs that conform with the 1989 version and studies of new medical devices that follow Helsinki's 1983 guidelines. ${ }^{32}$ Unfortunately, there is no mechanism, adjudicative or otherwise, for resolving ambiguities in interpretation and understanding. We hope that drafters of future revisions are able to create a document that is more carefully crafted and more ethically defensible. As Senn ${ }^{26}$ noted "those who draft guidelines that are meant to legislate for the behavior of others ought to pay more than a little care to the wording".

\section{References}

1 Ready T. Placebo trials deemed unethical. Nat Med 2000; 6: 1198.

2 Vastag B. Helsinki Discord? A controversial declaration. JAMA 2000; 284: 2983-85.

3 Lurie P, Wolfe SM. Unethical trials of interventions to reduce perinatal transmission of the human immunodeficiency virus in developing countries. N Engl f Med 1997; 337: 853-56.

4 Medical Ethics Committee of the World Medical Association. Proposed revision of the Declaration of Helsinki. Bull Med Ethics 1999; 150: revision

5 Brennan T. Proposed revisions to the Declaration of Helsinki-will they weaken the ethical principles underlying human research? $N$ Engl f Med 2000; 341: 527-30.

6 Anon. A fifth amendment for the Declaration of Helsinki. Lancet 2000; 356: 1123.

7 Lansang MA, Crawley FP. The ethics of international biomedical research. $B M F$ 2000; 321: 777-78.

8 World Medical Association website newsletter (www.wma.net/e/home.html, accessed June, 2001). 
9 Enserink M. Helsinki's new clinical rules: fewer placebos, more disclosure. Science 2000; 290: 418-19.

10 Council for International Organisations of Medical Sciences. International ethical guidelines for biomedical research involving human subjects. Geneva: CIOMS, 1993.

11 WHO. Operational guidelines for ethics committees to review biomedical research. Geneva: WHO, 2000.

12 ICH Harmonised Tripartite guideline: guideline for good clinical practice (available at www.ifpma.org)

13 UNAIDS guidance document. Ethical considerations in HIV preventive vaccine research. New York: UNAIDS, 2000.

14 Brown D. Medical research group revises guidelines on placebos. Washington Post, October 8, 2000: A2

15 Weijer C. Thinking clearly about research risk: implications of the work of Benjamin Freedman. IRB 1999; 21: 1-5.

16 Appelbaum PS, Roth LH, Lidz CW, Benson P, Winslade W. False hopes and best data: consent to research and the therapeutic misconception. Hastings Center Report, 1987; 17: 20-24.

17 Drazen JM, Koski G. To protect those who serve. N Engl f Med 2000; 343: $1643-45$.

18 Lo B, Wolf L, Berkeley A. Conflict-of-interest policies for investigators in clinical trials. N Engl f Med 2000; 343: 1616-20.

19 Evaluation of NIH implementation of Section 491 of the Public Health Service Act, mandating a program of protection for research subjects. Bethesda: Office of Extramural Research, National Institutes of Health, 1998.

20 Annas GJ, Grodin MA. Human rights and maternal-fetal HIV transmission prevention trials in Africa. Am f Public Hlth 1998; 88: 560-63.
21 National Bioethics Advisory Commission. Ethical and policy issues in international research (www.bioethics.gov)

22 Glantz LH, Annas GJ, Grodin MA, Mariner WK. Research in developing countries: taking "benefit" seriously. Hastings Center Report 1998; 28: 38-42.

23 Wendler D, Rackoff J. Informed consent and respecting individual autonomy: what's a signature got to do with it? IRB 2001; 23: 1-4.

24 National Institute of Health policy and guidelines on the inclusion of children as participants in research involving human subjects (http://grants.nih.gov/grants/guide/notice-files/not98-024.html accessed March, 1998).

25 Angell $M$. The ethics of clinical research in the third world. $N$ Engl $F$ Med 1997; 337: 847-49.

26 Senn S. The misunderstood placebo. Appl ClinTrials 2001; 10: 40-45,

27 Freedman B. Placebo-controlled trials and the logic of clinical purpose. $R B$ 1990; 12: $1-6$

28 Daniels N. Just health care. Cambridge: Cambridge University Press. 1985.

29 Marmot M, Wilkinson RG. Social determinants of health. Oxford: Oxford University Press, 1999.

30 Crouch RA, Arras JD. AZT trials and tribulations. Hastings Center Report, 1998; 28: 26-34.

31 Lie R. Ethical issues in clinical trial collaborations with developing countries - with special reference to preventive HIV vaccine trials with secondary endpoints. Presented at UNAIDS meeting on the ethics of HIV vaccine trials. May, 1999

32 US Department of Health and Human Services. Food and Drug Administration. Guidance for industry: acceptance of foreign clinical studies (www.fda.gov/cder/guidance/index.htm accessed March, 2001). 